\title{
Pediatric ANCA-Associated Vasculitis Presenting with Acute Postoperative Subglottic Stenosis: A Case Report
}

\author{
Diana T. Phan ${ }^{1}$, John Le ${ }^{1}$
}

\section{Abstract}

Introduction: Antineutrophil cytoplasmic antibody (ANCA)- associated vasculitis (AAV) often present with nonspecific constitutional symptoms. However, there has been increasing recognition of a subgroup of young, female AAV patients who are at higher risk for isolated subglottic airway disease.

Case Report: We report a rare case of a 17-year-old female with undiagnosed ANCA- associated vasculitis who presents with acute, life threatening subglottic stenosis requiring emergent intubation after routine surgery.

Conclusion: Much of the research on ANCA- associated vasculitis have been based on adult cases, however many pediatric patients may not follow the same diagnostic and clinical presentations. Clinicians should be vigilant in recognizing a subgroup of vasculitis patients who are often young, female, and have a higher prevalence of subglottic stenosis. Symptoms should be promptly worked up and initiation of immunosuppressive therapy is necessary to prevent life threatening complications and end-organ damage.

Keywords: ANCA- associated vasculitis; Subglottic stenosis; Granulomatosis with polyangiitis.

\section{Introduction}

Antineutrophil cytoplasmic antibody (ANCA)- associated vasculitis (AAV) is a group of disorders characterized by inflammation of blood vessels. It is categorized by factors such as vessel size, type of ANCA, pattern of organ involvement, and presence of granulomatous lesions [1]. AAV can present with nonspecific constitutional symptoms, and the diagnosis should be suspected in patients with multisystemic disease that is not caused by an infectious or malignant process [1].

The majority of AAV cases consist of two closely related entities: microscopic polyangiitis (MPA) and granulomatosis with polyangiitis (GPA). GPA characteristically involves granulomatous inflammation that can lead to gradual or acute airway obstruction [2]. Over $90 \%$ of patients with GPA present with upper and lower respiratory tract symptoms such as rhinitis, sinusitis, nasal ulcers, epistaxis, or erosive cartilage disease, and $20 \%$ can go on to develop subglottic stenosis (SGS) [2, 3]. Meanwhile, MPA more notably has renal involvement in up to $90 \%$ of patients and rarely involves granulomatous lesions [1].
There has been increasing recognition of a subgroup of GPA patients who exhibit unique characteristics including limited disease presentation (with less severe systemic findings), younger age, predominantly female, and higher frequency of SGS [2].

In this case report, we highlight the difficulty in diagnosing and managing $A A V$ in a pediatric patient who presents with acute SGS requiring emergent intubation after routine surgery.

\section{Case Report}

A 17-year-old otherwise healthy female was evaluated in otolaryngology clinic for chronic laryngitis, frequent epistaxis, and worsening dysphonia. She denied exposure to secondhand smoke, substance use, and overuse of her voice (e.g. singing, screaming). She had trialed antibiotics and steroids without improvement, and on exam she had dysphonia and signs of chronic inflammation of her bilateral tonsils. Flexible laryngoscopy revealed chronic laryngitis with an otherwise normal airway. She returned to clinic five months later with persistent dysphonia, increased nasal

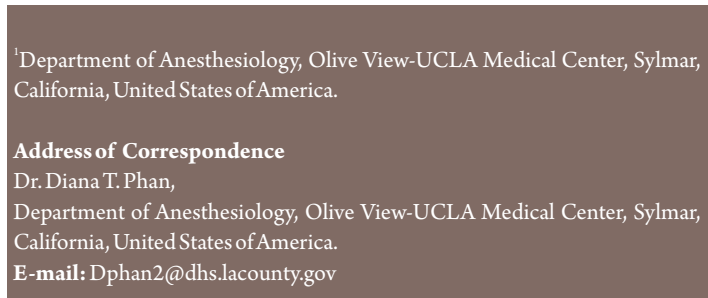

${ }^{1}$ Department of Anesthesiology, Olive View-UCLA Medical Center, Sylmar,

California, United States of America.

Address of Correspondence

Dr. Diana T.Phan,

California, United States of America.

E-mail: Dphan2@dhs.lacounty.gov

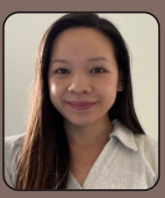

Dr. Diana T. Phan

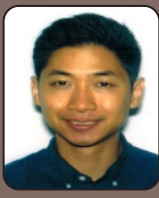

Dr. John Le

2021 (C) Journal of Anaesthesia and Critical Care Case Reports| Available on www.jaccr.com | ISSN 2454-7174| DOI: 10.13107/jaccr.2021.v07i01.168

This is an Open Access article distributed under the terms of the Creative Commons Attribution Non-Commercial License (http://creativecommons.org/licenses/by-nc/3.0) which permits unrestricted non-commercial use, distribution, and reproduction in any medium, provided the original work is properly cited.

14 | Journal of Anaesthesia and Critical Care Case Reports | Volume 7 | Issue 1 | January-April 2021 | Page $14-17$ 


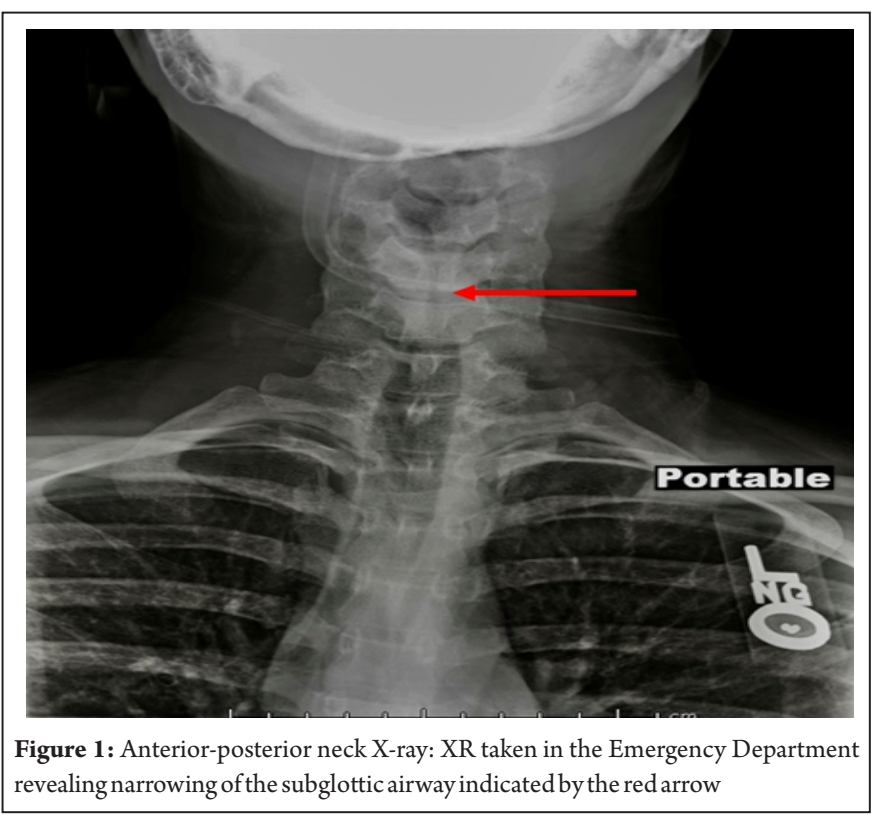

discharge, and a new onset cough. A repeat laryngoscopy revealed false vocal cord and glottic edema suggestive of chronic laryngeal irritation and dysfunction. She was treated for chronic sinusitis and post-nasal drip, which was thought to be contributing to her laryngeal irritation. She was then scheduled for tonsillectomy with balloon sinuplasty.

On the day of surgery, she was dysphonic but without respiratory distress. Physical exam showed normal vital signs, saturation $100 \%$ breathing room air, lungs clear to auscultation, normal airway anatomy except for tonsillar hypertrophy, Mallampati 2 exam, and body mass index (BMI) 23. General anesthesia was induced in a standard fashion with midazolam, fentanyl, lidocaine, propofol, and rocuronium. Mask ventilation was easy and a 7.0 cuffed oral endotracheal tube (ETT) was placed under direct visualization without complications or resistance. She then underwent an uncomplicated tonsillectomy. When examining the nasal cavities, the surgeons noted severely inflamed and friable mucosa, pus in the ostiomeatal complex, and polypoid changes without ulceration. A left balloon sinuplasty was done and tissue samples were obtained. At the end of surgery, she was extubated without complications after full reversal of neuromuscular blockade. Her hospital recovery course was uneventful, and she was discharged home four hours after surgery without any respiratory complaints.

Nine hours later, she presented to the Emergency Department with progressive shortness of breath. On exam, the patient exhibited stridor and her oxygen saturation was $92 \%$. Chest x-ray showed no acute pulmonary process, but neck $x$-ray showed diffuse narrowing of the subglottic airway (Fig. 1). Flexible laryngoscopy revealed normal epiglottis and vocal cords, but severe circumferential subglottic narrowing to $3-4 \mathrm{~mm}$. She was placed on supplemental oxygen at 15 liters per minute by facemask and given intravenous dexamethasone, nebulized racemic epinephrine, and intramuscular epinephrine for suspected anaphylaxis. She was then transferred to the intensive care unit for acute respiratory distress and was emergently intubated using a video laryngoscope. Subglottic resistance was noted during placement of a 5.5 cuffed ETT.

Given the constellation of findings, rheumatology consultation was obtained for workup of vasculitis and she was started on corticosteroids. On postoperative day three, a cuff leak was confirmed and she was taken to the operating room for a controlled extubation. Under direct visualization, the subglottic edema was confirmed to have resolved, and granulation tissue noted in the infra and subglottic regions were treated with intralesional triamcinolone. She was then successfully extubated without complications.

Histopathology of the tonsils revealed reactive follicular hyperplasia and Actinomyces. The nasal mucosal biopsy showed nonspecific chronic rhinosinusitis and fibrosis. Laboratory workup revealed positive antinuclear antibody (ANA), positive ANCA, positive myeloperoxidase (MPO), and negative proteinase 3 (PR3). On the basis of these findings, she was started on treatment for AAV with rituximab and oral corticosteroids. At three- and six-month follow up, she had no dysphonia or dyspnea, normal laryngoscopy exams, and her disease was well controlled.

\section{Discussion}

This case is a rare example of pediatric $A A V$ presenting with life threatening SGS after routine surgery. The nondescript preoperative symptoms of mild upper airway complaints made the diagnosis difficult to differentiate from common postnasal drip and sinusitis, and the concern for AAV arose after the patient developed acute postoperative SGS. The diagnosis of GPA was then made on the basis of the patient's serologies and clinical presentation. Although biopsies containing granuloma or vasculitis are helpful to confirm diagnosis, they can often be non-specific with chronic inflammation- such was the case with our patient's surgical pathology $[1,3]$. The type of ANCA can also help with classification because PR3-ANCA is more commonly associated with GPA, and MPO-ANCA is more frequent in MPA [2, 4]. Our patient had MPO-ANCA despite our high clinical suspicion for GPA. Although infrequent, studies have shown that up to $25 \%$ of GPA and MPA patients may have the alternative ANCA $[1,2,5]$.

There has been increasing recognition of the subset of MPOANCA GPA patients similar to ours. Though previous studies acknowledge that GPA can affect patients at any age, most report that the common age of onset is 40 years and is 
more common in men [1]. Distinctly, MPO-ANCA GPA patients are often younger and predominantly female, more often have limited disease without severe organ involvement, and have a higher prevalence of SGS [2,6]. Similarly, a study of 960 GPA patients found that those with SGS were significantly more likely to be female ( $72 \%$ vs. $53 \%$ ), younger (36 vs. 49 years old), and less likely to have renal involvement (39\% vs. 62\%). Even during acute episodes of SGS, over 50\% of these patients only had disease limited to the upper airway [7]. From these findings, it has been suggested that MPOANCA GPA patients should be identified as a unique subgroup of AAV.

It is also important to note that $\mathrm{AAV}$ is rare in the pediatric population. The estimated incidence of GPA in adults is $1: 100,000$, while in children (age $<18$ years old) it is $1: 1,000,000$. GPA is the most common AAV in pediatric groups and only $3 \%$ of cases are diagnosed before the age of 20. There are few studies on pediatric AAV due to the rare occurrence in childhood, but several have shown a female predominance in the pediatric population compared to adults $[8,9]$. Pediatric GPA patients will often present with upper airway and constitutional symptoms, but they can also display severe disease both at presentation and during the disease course. One study reported over $80 \%$ of children with GPA required hospitalization at the time of diagnosis, and $50 \%$ of them required intensive care for mostly ventilator support or dialysis [9]. Though GPA can present with similar manifestations in children and adults, disease severity and organ involvement may differ. Much of the literature on AAV is based on adult cases and more research is needed to better characterize the presentation and clinical course in children $[8,9]$.

This case demonstrates the difficulty of diagnosing AAV and specifically highlights the challenge with pediatric MPOANCA GPA patients who may present with SGS. This presentation of GPA is in contrast to the previously accepted "classic triad" of upper respiratory, pulmonary, and renal findings $[1,6]$. Symptoms concerning for SGS, such as dysphonia, hoarseness, stridor, and dyspnea should be promptly evaluated. Clinicians should have a high suspicion for SGS in young, female patients with GPA, especially those with isolated upper airway disease. In many cases, initiation of treatment with immunosuppressive therapy is necessary to prevent life threatening complications and end-organ damage. Nearly half of patients with SGS may need surgical intervention in addition to immunosuppression [2]. These patients need close follow-up by a multidisciplinary care team including specialists in Rheumatology, Otolaryngology, and possibly Nephrology.

\section{Conclusion}

Clinicians must be vigilant in recognizing patients with possible AAV who may not fulfill the common diagnostic criteria or classifications, especially in young, female patients.

\section{Clinical Relevance}

1. AAV can present with nonspecific constitutional symptoms, and the diagnosis should be suspected in patients with multisystemic disease not caused by infectious or malignant processes.

2. MPO-ANCA GPA patients should be identified as a unique subgroup; they are often younger, female, and have a higher prevalence of SGS.

3. Much of the literature on AAV is based on adult cases and more research is needed to better characterize the presentation and clinical course in children.

4. Symptoms concerning for SGS should be promptly evaluated. Prompt initiation of immunosuppressive therapy is necessary to prevent life threatening complications and end-organ damage from AAV.

\section{References}

1. Mansi IA, Opran A, Rosner F. ANCA-associated small-vessel vasculitis. Am Fam Physician 2002;65:1615-20.

2. Schirmer JH, Wright MN, Herrmann K, et al. Myeloperoxidaseantineutrophil cytoplasmic antibody (ANCA)-positive granulomatosis with polyangiitis (Wegener's) is a clinically distinct subset of ANCAassociated vasculitis. Arthritis Rheum 2016;68:2953-2963.

3. Blackabey V, Gan RW, Buglass $H$, et al. Granulomatosis with polyangiitis causing subglottic stenosis: two cases and their management. AME Case Rep 2018;2:17.

4. Mohammad AJ, Mortensen KH, Babar J, et al. Pulmonary involvement in antineutrophil cytoplasmic antibodies (ANCA)-associated vasculitis: the influence of ANCA subtype.J Rheumatol 2017;44:1458-1467.
5. Preto C, Silva A, Alves S, et al. Case Report: The diagnosing challenge of a positive ANCA Vasculitis in the Paediatric Age. Hindawi Case Reports in Pediatrics 2017;

Article ID 2962794.https://doi.org/10.1155/2017/2962794.

6. Marroquin-Fabian E, Ruiz N, Mena-Zuniga J, et al. Frequency, treatment, evolution, and factors associated with the presence of tracheobronchial stenoses in granulomatosis with polyangiitis. Retrospective analysis of a case series from a single respiratory referral center. Seminars in Arthritis and Rheumatism 2019;48:714-719.

7. Quinn KA, Gelbard A, Sibley C, et al. Subglottic stenosis and endobronchial disease in granulomatosis with polyangitis. Rheumatology 2019;58:2203-2211. 
8. Bohm M, Gonzalez Fernandez MI, Ozen S, et al. Clinical features of childhood granulomatosis with polyangiitis (wegener's granulomatosis). Pediatr Rheumatol Online J2014;12:18.
9. James KE, Xiao R, Merkel PA, et al. Clinical course and outcomes of childhood-onset granulomatosis with polyangiitis. Clin Exp Rheumatol 2017;35 (Suppl 103): 202-208

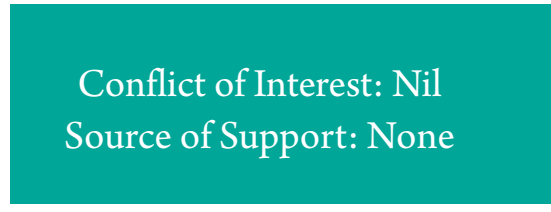

\section{How to Cite this Article}

Phan DT, Le J | Pediatric ANCA- Associated Vasculitis Presenting with Acute Postoperative Subglottic Stenosis: A Case Report | Journal of Anaesthesia and Critical Care Case Reports | January-April 2021; 7(1): 14-17. 